\title{
The introduction of genetically modified microorganisms designed for rhizoremediation induces changes on native bacteria in the rhizosphere but not in the surrounding soil
}

\author{
Daniel Aguirre de Cárcer ${ }^{1}$, Marta Martín¹, Martina Mackova ${ }^{2,3}$, Thomas Macek ${ }^{2,3}$, \\ Ulrich Karlson ${ }^{4}$ and Rafael Rivilla ${ }^{1}$ \\ ${ }^{1}$ Departamento de Biología, Universidad Autónoma de Madrid, Madrid, Spain; ${ }^{2}$ Department of Natural \\ Products, Institute of Organic Chemistry and Biochemistry, CAS, Prague, Czech Republic; ${ }^{3}$ Department of \\ Biochemistry and Microbiology, Faculty of Food and Biochemical Technology, ICT Prague, Prague, Czech \\ Republic and ${ }^{4}$ National Environmental Research Institute, University of Aarhus, Roskilde, Denmark
}

\begin{abstract}
A 168-day microcosms experiment was used to assess the possible functional and structural shifts occurring in the bacterial community of a site with a historical record of polychlorinated biphenyl (PCB) contamination, after the introduction of plants whose roots have been inoculated with genetically modified (GM) microorganisms, designed for rhizoremediation. Salix sp. plants were inoculated with two different GM Pseudomonas fluorescens strains or with their parental wild-type strain. Both bulk soil and rhizosphere samples were analyzed. Physiological profiles based on 31 ecologically relevant carbon sources were used to detect differences in bacterial community functions. The community structure of eubacteria, $\alpha$ and $\beta$-proteobacteria, actinobacteria and acidobacteria communities were analyzed via a polymerase chain reaction-thermal gradient gel electrophoresis (TGGE) approach. The introduced transgenes had no effect on the function and structure of the bacterial community in bulk soil, although they enhanced biodegradation of PCBs as determined by chemical analysis. However, the transgenes effected the development of functionally and genetically distinct bacterial communities in the rhizosphere. Moreover, structural and functional differences were detected between planted and unplanted soils and between soil and rhizosphere samples. In the case of the different group-specific structures studied, differences were observed between groups because of time-dependant shifts, rhizosphere effect and bacterial strain introduced.
\end{abstract}

The ISME Journal (2007) 1, 215-223; doi:10.1038/ismej.2007.27

Subject Category: microbe-microbe and microbe-host interactions

Keywords: CLPP; GMO; impact; PCBs; rhizoremediation; TGGE

\section{Introduction}

Polychlorinated biphenyls (PCBs) have been detected in most ecosystems, especially in soils and sediments of industrial areas (McFarland and Clarke, 1989). Such ubiquitous presence is explained by their high chemical stability and low water solubility, which contribute to their persistence in the environment, but also by the fact that more than 300 million $\mathrm{kg}$ have been released in the

Correspondence: Dr R Rivilla, Departamento de Biología, Universidad Autónoma de Madrid, Darwin 2, Campus de Cantoblanco, Madrid 28049, Spain.

E-mail: rafael.rivilla@uam.es

Received 29 December 2006 revised and accepted 21 March 2007 environment before their use was abandoned in most countries (Holoubek, 2001). PCBs accumulate in higher trophic levels of the food chain and cause numerous toxic effects on different organisms (Safe, 1994). Thus, their persistence causes a major environmental problem. Remediation of PCB-contaminated soil has traditionally been carried out by incineration or burial of the soil in secure landfills. As a less expensive removal strategy, rhizoremediation (Yee et al., 1998), the use of a dual plantmicroorganism system, in which the plant provides nutrients, support and a greater availability of the substrate, and the microorganisms drive the enzymatic remediation, has been proposed (Kuiper et al., 2004; Villacieros et al., 2005). Salix sp., which produces salicylic acid and related compounds that 
induce the degradation of many xenobiotic molecules (such as polycyclic aromatic hydrocarbons) and sustain bacterial growth (Singer et al., 2003; Leigh et al., 2006) are good candidates for rhizoremediation of PCB-contaminated soils. Since the desired biotransformation abilities and the aptitude to thrive in rhizosphere environments are seldom observed together in any given strain, the use of genetically modified (GM) microorganisms (GMMs) is being considered. Before using such GMMrhizoremediation technologies for the treatment of polluted sites, risk assessments are required by law. Such risk assessments normally concern (as in the EU Directive 2001/18/EC) the persistence and dissemination potential of the GMM, the potential transfer of inserted genetic material to indigenous organisms and the impact of the GMM on native microorganisms (Drobnik, 1999). Thus, approval for field testing will normally be dependant on the results of studies conducted in simulated natural environments (Morrissey et al., 2002). The aim of this microcosm study was to evaluate the possible functional and structural shifts occurring in the bacterial populations of a PCB-contaminated soil ecosystem after the introduction of integrated plantGMM systems designed for PCB-rhizoremediation and compare them with the introduction of the wild-type parental microorganism. Our results show that the impact of the introduced transgenes on the different bacterial populations studied was limited to the rhizosphere of the inoculated plants.

\section{Materials and methods}

\section{Experimental setup}

Bacteria were grown overnight in liquid cultures on sucrose asparragine (SA) medium and washed in phosphate buffer. Willows (Salix viminalis $x$ schwerinii, variety Björn), were pregrown in tap water from 20-cm-long cuttings. The root systems of 3-week-old willow plants were submerged in phosphate buffer containing $10^{6}$ bacteria/ml for $1 \mathrm{~h}$. The bacterial treatments were as follows: control (Pseudomonas fluorescens F113rif; wild-type strain with a spontaneous rifampicine resistance (Fedi et al., 1996)), class 1 GM strain (P. fluorescens F113rifpcb; single chromosomal insertion of the $b p h$ operon (Brazil et al., 1995)) and class 2 GM strain ( $P$. fluorescens F113L::1180; single chromosomal insertion of the bph operon under the control of the Sinorhizobium meliloti nod regulatory system, which shows increased growth rate on biphenyl (Villacieros et al., 2005)). Three inoculated willow plants were planted per galvanized iron pot on $1900 \mathrm{~g}$ of previously homogenized and sieved $(2 \mathrm{~mm}$ mesh) PCB-contaminated soil. The soil, obtained from Lhenice (Czech Republic), was contaminated with approximately $200 \mathrm{mg} / \mathrm{kg}$ of the PCB mixture Delor103 (similar to Aroclor 1242) resulting from historic industrial activity. Controls contained uninoculated soil only. All pots were incubated under controlled conditions of $16 \mathrm{~h}$ light, at $22^{\circ} \mathrm{C}$ and $8 \mathrm{~h}$ dark, at $18^{\circ} \mathrm{C}$. All pots were watered from below. Three replicate pots per treatment were killed and analyzed for each sampling date. Bulk soil samples (from a total of 12 pots per sampling dates) were taken as soils cores at seven sampling dates, starting with day 0 . Rhizosphere samples (from a total of nine pots per sampling dates), defined as roots plus the soil still adhering to the roots after consistent shaking, were taken every 4 weeks for 6 months (six sampling dates). Soil and rhizosphere samples from each pot were used for the community-level physiological profiles (CLPPs) statistical analysis. Soil and rhizosphere-derived DNA was obtained from each pot and then soil and rhizospheric DNA samples from the same treatments were pooled. Thus, each final genetic profile (one profile per time and treatment) was derived from the combined DNA samples from three different pots with the same treatment. To document the persistence of the inocula, triplicate rhizosphere samples were analyzed at all seven sampling times by extracting in phosphate buffer and plating on SA plates containing rifampicin (colonies were doublechecked by morphological appearance), which resulted in an inoculum presence that ranged between $10^{5}$ and $10^{6} \mathrm{CFU} / \mathrm{g}$ rhizosphere wet weight for all inocula during the first 91 days and declined to $10^{4} \mathrm{CFU} / g$ on day 163 .

\section{PCB analysis}

Triplicate soil samples from each sampling date and treatment were air-dried for $24 \mathrm{~h}$ at room temperature, then homogenized with mortar and pestle and sieved through a 1-mm mesh. Aliquots of soil $(1 \mathrm{~g})$ were extracted in hexane for $4 \mathrm{~h}$ using a micro-scale modification of the EPA method for Soxhlet extraction (EPA Method 3540C; Ryslava et al., 2003). Extracts were concentrated under a nitrogen stream and subjected to florisil cleanup (EPA Method 3620B) before analysis. Each sample was analyzed in duplicate using a Hewlett-Packard 5890 gas chromatograph with an electron capture detector and a fused silica capillary column $(30 \mathrm{~m}, 0.20-\mathrm{mm}$ inner diameter) coated with $0.25-\mu \mathrm{m}$ stationary phase SE054 with nitrogen as the carrier gas (flow rate $1 \mathrm{ml} / \mathrm{min}$ ). The total PCB concentration was estimated based on indicator congeners using US EPA methods 8089/8081 for expressing the total content of PCBs as a sum of recommended indicator congeners (PCB no. 28-2,4,4'-trichlorobiphenyl, 52-2,2',5,5'-tetrachlorobiphenyl, 101-2, $2^{\prime}, 4,5,5^{\prime}$-pentachlorobiphenyl, 138-2,2',3,4,4',5-hexa-

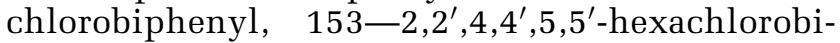
phenyl, 180-2, $2^{\prime}, 3,4,4^{\prime}, 5,5^{\prime}$-heptachlorobiphenyl). The effect of the different treatments on PCB concentration was assessed by analysis of variance employing general linear modelling (GLM) using the SPSS 14.0 software. 


\section{CLPP}

Soil samples $(5 \mathrm{~g})$ from each replicate pot were placed in $250 \mathrm{ml}$ flasks containing $45 \mathrm{ml}$ sterile saline solution and placed in an orbital shaker at 250 r.p.m. for $30 \mathrm{~min}$ at $28^{\circ} \mathrm{C}$. Rhizosphere samples $(0.5 \mathrm{~g})$ were vortexed for $2 \mathrm{~min}$ in $4.5 \mathrm{ml}$ of sterile saline solution. Soil and root debris was removed by a low-speed centrifugation step ( $2 \mathrm{~min}, 500 \mathrm{~g}, 20^{\circ} \mathrm{C}$ ). The bacterial fraction was harvested from the supernatant by centrifugation at $1000 \mathrm{~g}$ for $20 \mathrm{~min}$. The resulting bacterial pellet was then washed in $10 \mathrm{ml}$ of sterile saline solution to avoid carry-over of soil-carbon sources to the microplate and resuspended in $10 \mathrm{ml}$ of saline solution. Each suspension of bacterial cells was diluted 1:10 and used to inoculate ECOplates (Biolog, CA, USA), $135 \mu \mathrm{l}$ per well, which were then incubated at $28^{\circ} \mathrm{C}$ in darkness. Each ECOPlate contains 31 response wells with different soil-carbon sources and a control well without carbon source. A tetrazolium redox dye is included, which turns purple when reduced by microbial respiration. The $\mathrm{OD}_{595}$ was measured using a Spectra Fluor microtiter plate reader (Tecan, Zurich, Switzerland) after 22, 44, 66 and $88 \mathrm{~h}$. The raw $\mathrm{OD}$ values in each microplate were corrected for the background color in the control well without carbon source. To account for slightly differing initial cell densities, the corrected ODs were divided by the average well color of the plate, giving the standardized OD, thus comparing standardized patterns rather than absolute values (Garland and Mills, 1991). The activity values obtained when a maximum of wells showed activity (66 h) (Glimm et al., 1997) were chosen for further statistical analysis. Activity values smaller than 0.006 only contributed to noise and were counted as 0 (no catabolic activity). The resulting 31 variables (corresponding to each carbon source) were reduced to seven new principal components (SPSS 12.0), which in all cases explained more than $75 \%$ of the total variance (total eigenvalue sum), using the covariance of the variables. To test for differences between the communities, a multivariate analysis was performed (GLM, SPSS12.0) using the new principal components as variables (Glimm et al., 1997). Diversity of the catabolic profiles was calculated using the Shannon index (Shannon and Weaver, 1963) according to the following formula: $H=-\Sigma P i \log P i$, where $P i$ represents the proportion of each particular activity value.

\section{DNA extraction and PCR amplification of group-} specific $16 S$ rDNA

Total DNA was extracted from $0.5 \mathrm{~g}$ (wet weight) of each of the replicate samples following a protocol described by Porteous et al. (1997). Owing to the presence of contaminating humic material, the DNA was further purified using a Microcon YM-100 filtering device (Millipore, Bedford, MA, USA). The size and quality of the DNA was then checked on agarose gels. One microliter of pooled DNA ( $\sim 15 \mathrm{ng}$ ) was used to amplify the 16S rRNA genes with group-specific primers targeting eubacteria $(27 \mathrm{f}$ (Weisburg et al., 1991) and L1494 (Weisburg et al., 1991)), $\alpha$-proteobacteria ( $\alpha$-U203 (Gomes et al., 2001) and L1494), $\beta$-proteobacteria (F948 $\beta$ (Gomes et al., 2001) and L1494), acidobacteria (31F (Barns et al., 1999) and L1494) and actinobacteria (F243HGC (Heuer et al., 1997) and R1378 (Heuer et al., 1997)). The polymerase chain reaction (PCR) mixture consisted of $2.5 \mu \mathrm{l}$ of reaction buffer, $5 \mathrm{pmol}$ of primers, $3.75 \mathrm{mM} \mathrm{MgCl}_{2}, 0.2 \mathrm{mM} \mathrm{2}$-deoxyribonucleoside $5^{\prime}-O$-(1-thiotriphosphate)s, $5 \mu \mathrm{g}$ bovine serum albumin, $4 \%$ dimethylsulfoxide and $1 \mathrm{U} \mathrm{T} t h$ DNA polymerase (Biotools, Madrid, Spain), in a final volume of $25 \mu \mathrm{l}$. The PCR program was as follows: 5 min denaturation at $94^{\circ} \mathrm{C}$ followed by 25 cycles of $1 \mathrm{~min}$ at $94^{\circ} \mathrm{C}, 1 \mathrm{~min}$ for primer annealing, $2 \mathrm{~min}$ at $72^{\circ} \mathrm{C}$ for primer extension and a final cycle at $72{ }^{\circ} \mathrm{C}$ for $10 \mathrm{~min}$. The products of three replicate PCR reactions were then pooled. One microliter of a 1:10 dilution of these amplified products was used for a second round of nested PCR using high-performance liquid chromatography-purified (Villadas et al., 2002) primer pair F984GC (Heuer et al., 1997) and R1378. Acetamide was added to the reaction mixture to facilitate the denaturation of dsDNA and to circumvent the formation of secondary structures. This second PCR results in 475 bp fragments (968-1401; 16S rRNA Escherichia coli numbering) suitable for thermal gradient gel electrophoresis (TGGE) analysis.

\section{TGGE}

For sequence-specific separation of the PCR products, a TGGE system (Biometra, Göttingen, Germany) was used as specified by the manufacturer. Polyacrylamide gels $(6 \%)$ were polymerized in $1 \times$ TAE buffer, $8 \mathrm{M}$ urea, $20 \%$ deionized formamide and $2 \%$ glycerol. PCR samples $(8.5 \mu \mathrm{l})$ were resolved by electrophoresis in $1 \times$ TAE buffer at $130 \mathrm{~V}$, through a temperature gradient of $44.5-56^{\circ} \mathrm{C}$ during $16 \mathrm{~h}$. Reference patterns consisting of various groupspecific sequences were included in the gels to facilitate the gel analysis. DNA gel bands were evidenced by a silver-staining protocol (Heuer et al., 1997).

\section{Data analysis}

The gels were digitally documented and analyzed (Quantity One, Bio-Rad, Hercules, CA, USA). After lane-based background subtraction employing the rolling disk method, bands were detected and the resulting banding patterns were standardized with the reference pattern included. Each band was described by its standardized position and its relative intensity in the profile, which was calculated by the relative surface of the peak in the profile over the sum of the surfaces for all the peaks within 
the profile. The data obtained, relative abundances and positions were subjected to correspondence analysis (CA) (XLSTAT package), to represent the profiles in two dimensions and to observe their temporal change. CA is well adapted to abundance data since its underlying model assumes a unimodal distribution of species along environmental gradients (as stated by Niche theory) and since it uses $\chi^{2}$ distances, thus avoiding the double-zeros problem (Legendre and Legendre, 1998). Moreover, the data were subjected to hierarchical agglomerative clustering using the unweighted pair-group method using average linkages method (UPGMA), based on the whole-period averaged $\chi^{2}$ dissimilarities, and significant clusters in the dendrograms were determined by setting the automatic detect function (XLSTAT package). The GMM and unplanted soil treatments dissimilarities versus the control treatment were further studied by analysis of variance (SPSS 12.0) and diversity of the genetic profiles was calculated using the Shannon index (as above), where $\mathrm{Pi}$ represents the proportion of a particular band in the profile.

\section{Results}

\section{PCB degradation}

To determine the efficiency of the GM strains in PCB degradation under soil microcosm conditions, we measured the amount of PCB present initially and at each sampling time. The initial PCB concentration, measured as the sum of six recommended indicator congeners was $182 \pm 22 \mathrm{mg}$ PCB $/ \mathrm{kg}$ dry soil. After 168 days PCB concentrations were $186 \pm 31,164 \pm 8$ and $158 \pm 16 \mathrm{mg} \mathrm{PCB} / \mathrm{kg}$ dry soil, for the wild-type, class 1 and class 2 treatments, respectively. Statistical analysis of the data from the seven time points, showed significant differences between the wildtype treatment and class $1(P<0.013)$ and class 2 $(P<0.009)$ treatments. No significant differences were observed between class 1 and class 2 treatments.

\section{Analysis of the structural changes in the eubacteria community}

To observe differences in the structure of the microbial communities studied, genetic profiles were generated by PCR amplification of the $16 \mathrm{~S}$ rRNA genes present in the DNA obtained from each sample, followed by sequence specific separation via TGGE (Figure 1). The average dissimilarities between the genetic profiles derived from the different treatments were represented in dendrograms (Figure 2) to assess the overall effect of the treatments on the structure of the community. In addition, to observe the temporal changes in the structure of the communities, the genetic profiles were subjected to CA (Figure 3). In the dendrogram derived from the eubacterial genetic profiles (Figure 2a), the different treatments were grouped

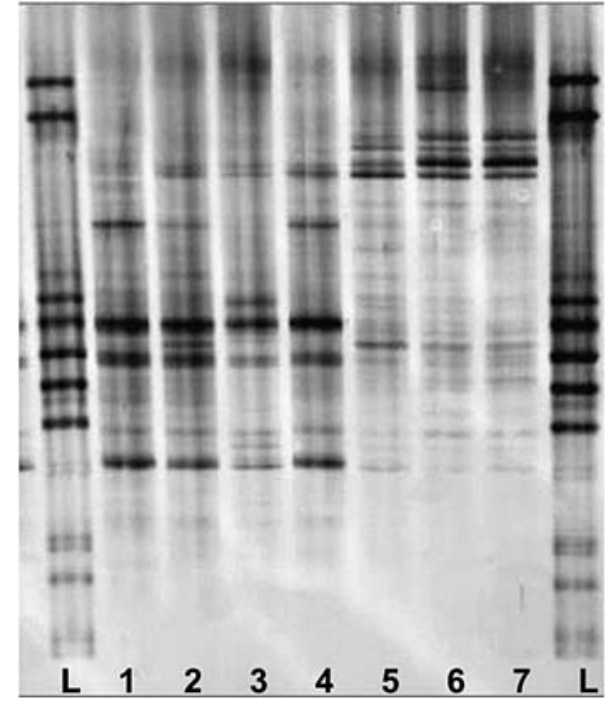

Figure 1 Example of TGGE banding patterns corresponding to the $\alpha$-proteobacterial patterns at sampling date 2 (28 days). L, group-specific ladder. 1-7, treatments: unplanted soil, Wt soil, class 1 soil, class 2 soil, Wt rhizosphere, class 1 rhizosphere and class 2 rhizosphere.

in four significant clusters (dashed lines), which were formed by the four bulk soil samples in a single cluster and the three-rhizosphere samples in separate clusters. Similar results can also be observed in the CA plot (Figure 3a); there was a clear separation between rhizosphere and soil profiles, which grew with time. The soil profiles showed a low dispersion because of treatment or sampling date, while the rhizosphere profiles showed a higher dispersion, with marked temporal shifts. The class 2 rhizosphere profiles were very different from the control profiles, while the class 1 profiles were intermediate, oscillating between being similar to the control or to the class 2 profiles at different time points. Thus, no evident effect was observed in the soil eubacterial structure because of treatment. Conversely, the treatments had noticeably different effects on the development of the rhizosphere eubacterial community structure.

\section{Analysis of the structural changes in specific} populations

When the same approach was used to analyze the structural changes of specific bacterial populations $(\alpha$ and $\beta$-proteobacteria, acidobacteria and actinobacteria), similar results were obtained. As in the case of the eubacterial profiles, the data grouped in four clusters for all the populations analyzed with the exception of the acidobacteria (Figure 3). These four clusters were formed by the soil samples in one cluster and the three-rhizosphere samples in separate clusters. The dissimilarity values between soil profiles were low, while the genetic profiles from the different rhizosphere treatments usually were very dissimilar. The CA plots (Figure 3) also showed 


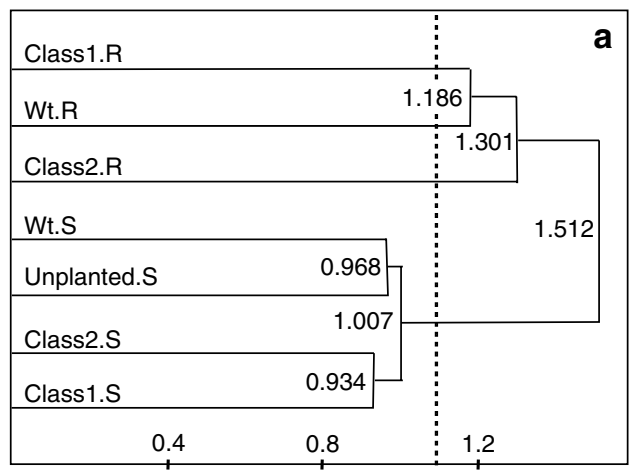

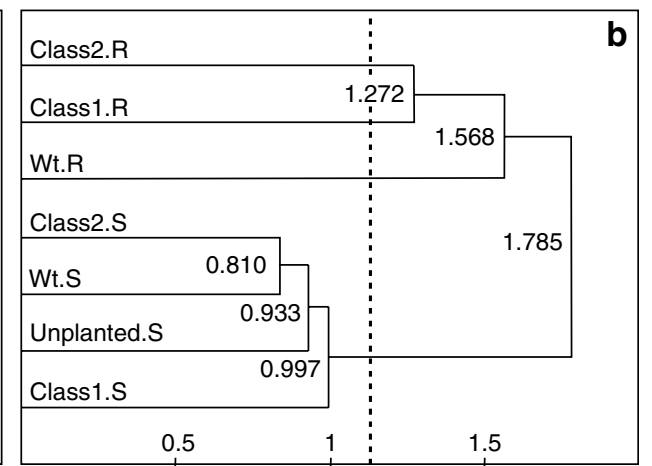

\begin{tabular}{|c|c|c|c|}
\hline Class2.R & & & C \\
\hline Class1.R & & 1.614 & \\
\hline Wt.S & & & \\
\hline Unplanted.S & 0.915 & 1.671 & \\
\hline Class2.S & 1.092 & & \\
\hline Class1.S & 1.189 & 1.760 & \\
\hline Wt.R & & & \\
\hline 0.5 & 1 & 1.5 & \\
\hline
\end{tabular}

\begin{tabular}{|c|c|c|}
\hline Class1.S & & d \\
\hline Wt.S & 0.935 & \\
\hline Class2.S & 1.080 & \\
\hline Unplanted.S & 1.125 & \\
\hline Class1.R & & \\
\hline Wt.R & & 1.605 \\
\hline Class2.R & & 1.619 \\
\hline 0.5 & 1 & 1.5 \\
\hline
\end{tabular}

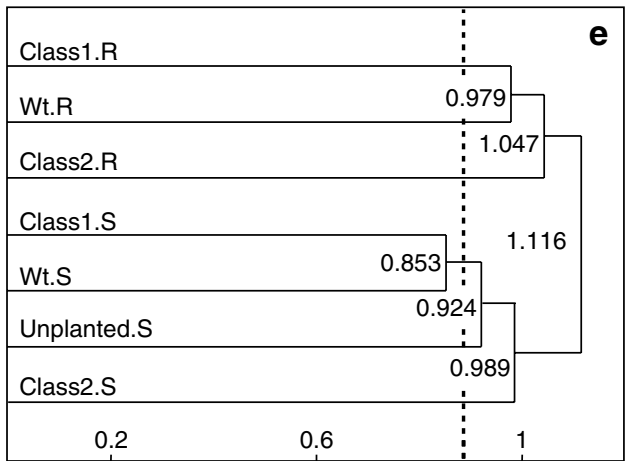

Figure 2 Dendrograms based on the whole-period averaged dissimilarities between soil (S) and rhizosphere (R) bacterial communities derived from the genetic profiles of the different treatments: Wt, F113rif. Class1, F113rifpcb. Class2, F113L::1180. Unplanted; unplanted soil. Numbers at nodes and abscissa are $\chi^{2}$ dissimilarities. The dashed line represents cutoff for significant clusters. (a) Eubacteria, (b) $\alpha$-proteobacteria, (c) $\beta$-proteobacteria, (d) actinobacteria, (e) acidobacteria.

a clear separation between rhizosphere and soil profiles for all populations studied, which usually grew over time. In most cases, there was a low dispersion between soil profiles from different treatments. On the contrary, the rhizosphere profiles were more dispersed, with marked temporal shifts (except in the case of $\alpha$-proteobacteria). However, there were some noticeable differences between populations. In the case of the $\alpha$-proteobacteria (Figure 3b), the difference between rhizosphere and soil profiles was very high from the first sampling date and no temporal changes were evident. For this population, both GMM treatments produced rhizosphere profiles that were very similar along time, while a steady difference was observed between the rhizosphere profiles produced by the GMM treatments and the rhizosphere profiles produced by inoculation with the wild-type strain.
In the $\beta$-proteobacteria CA plot (Figure 3c), the class 2 rhizosphere profiles were very different from the wild-type-produced profiles in two out of three sampling times, while the class 1 profile oscillated between that similar to the control (wild-type strain) or to the class 2 profile at different time points.

In the actinobacterial CA plot (Figure 3d), the differentiation between soil and rhizosphere profiles developed slowly and a low time-dependent drift was observed in the soil profiles. In the case of the acidobacteria (Figure 2e), dissimilarity levels between treatments were very low, indicating the absence of effect because of treatment, although an evident difference between soil and rhizosphere profiles can be observed. As for the actinobacteria, the differentiation between soil and rhizosphere acidobacterial profiles developed slowly (Figure 3e) and a low time-dependent drift was detected for the 

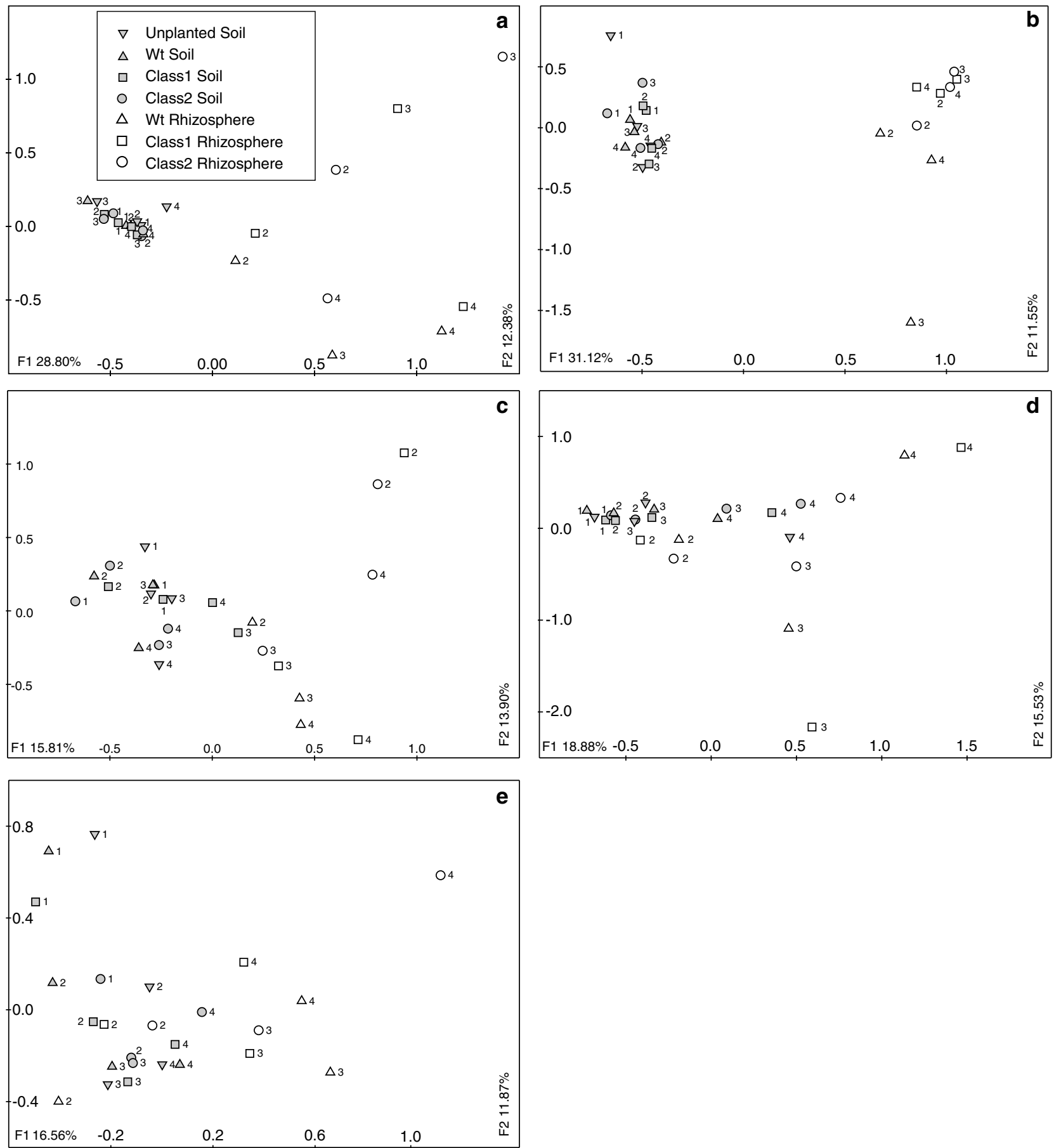

Figure 3 Correspondence analysis based on the genetic profiles of the bacterial communities from different treatments and sampling dates. Wt, F113rif. Class1, F113rifpcb. Class2, F113L:: 1180. Unplanted, unplanted soil. Numbers close to the objects represent sampling dates (1, 0 days; 2,28 days; 3,56 days; 4, 84 days). Values of the dimensions (\%) represent the percentage of the total variance explained by that dimension. (a) Eubacteria, (b) $\alpha$-proteobacteria, (c) $\beta$-proteobacteria, (d) actinobacteria, (e) acidobacteria.

soil profiles as well. The dispersion of the acidobacteria soil profiles at sampling dates 1 and 2 was surprisingly high, although it turned to a much less disperse distribution, similar to the other populations, at time points 3 and 4 . No statistical significant differences were found for all the groups studied comparing, by analysis of variance, the dissimilarities of the GMMs and unplanted soil treatments with respect to the control soil treatment, which means that the effect of the GMM treatments in the soil communities was not greater than the effect of leaving the soil unplanted. The Shannon indices of diversity based on the relative abundance of bands in the TGGE gels were tested by analysis of 
variance (not shown). However, no statistically significant differences owing to treatment or timedependent changes were detected either in soil or rhizospheric populations for all the different groups studied, based on Shannon indices of diversity.

Analysis of the function of the microbial community To assess possible differences between the functions of the microbial communities derived from different treatments and sampling dates, CLPPs were generated and analyzed. Statistically significant differences were detected between the CLPPs of the different sampling dates for all treatments $(P$-value 0.000), indicating that the function of the communities experienced a time-dependent drift. The CLPPs of the rhizospheric communities differed from those of their soil-counterparts ( $P$-value 0.002$)$, not surprisingly, taking into account the welldocumented rhizosphere effect. Moreover, the plants exerted an effect on the microbial function at a distance from the rhizosphere, since significant differences were also found comparing soil communities derived from planted and unplanted pots ( $P$-value 0.000). No significant differences were detected between the CLPPs of the soil communities with different treatments, which indicate that the function of the soil microbial communities was unaltered by the treatments. Conversely, in the case of the function of the rhizosphere communities, statistically significant differences were found between both class 2 ( $P$-value 0.012$)$ and class 1 $(P$-value 0.035$)$ treatments compared with the control, but no differences were observed between the class 2 and class 1 rhizosphere CLPPs. Such results indicate that both GMM treatments had an effect on the function of the rhizosphere communities that differed from that of the wild-type F113rif inoculation. The CLPPs were also used to obtain a measure of the functional diversity of the communities based on the Shannon index of diversity, which were then compared. The results indicate that the soil communities from planted pots were more diverse than those coming from unplanted pots (unplanted $2.91 \pm 0.18$; planted $3.03 \pm 0.11, P$-value 0.000$)$ and the rhizosphere communities were slightly more diverse than their soil counterparts (soil $3.03 \pm 0.11$; rhizosphere $3.08 \pm 0.11, P$-value 0.020$)$. These results, together with the precedent (see above), state that not only did the presence of Salix sp. plants alter the microbial function at a distance from the rhizosphere, but it made it more diverse as well. No differences were found between the catabolic diversity of soil communities with different treatments, but both class 2 (class $23.14 \pm 0.10$, control $3.08 \pm 0.11, P$-value 0.017$)$ and class $1(3.16 \pm 0.05$, $P$-value 0.001) treatments showed more diversity than the control treatment in the rhizosphere communities. No differences were found between class 1 and class 2 treatments.

\section{Discussion}

In the present study, we show that the introduction of two bacterial strains with genetically engineered traits for the degradation of PCBs in the rhizosphere of willow plants had no effect, compared with the introduction of the wild-type bacteria, on the surrounding PCB-contaminated soil microbial populations. This was evidenced by the fact that no statistically significant differences were detected between the CLPPs of different treatments (as well as between the diversity indices derived from them), even though such approach detected time-dependent changes and overall differences between planted versus unplanted soil communities and between rhizosphere versus soil communities. Similar results were obtained when the structure of the communities was analyzed; the soil genetic profiles were homogeneous, as evidenced by the small dispersion on the CA plots of most groups studied. Moreover, the fact that the clustering method applied grouped all bulk soil samples together, and the evidence that the effect of the GMMs treatments was not significantly greater than the effect caused by the lack of willow roots in the environment, support the conclusion that the soil communities were not noticeably affected by the difference in treatment (except possibly the $\beta$ proteobacterial structure, see below).

However, the introduction of such GMMs seems to have influenced the development of distinct rhizospheric communities, since the CLPPs of the GMMs treatments differed from those of the wild type. The genetic profiles were also different, which can be observed not only in the distinctive clusterings, but also in the dispersion observed in the CA plots. A similar picture can be drawn in the case of the group-specific analysis. There were some differences, for instance, regarding the time-dependent shifts of the profiles or the strength of the rhizosphere effect, which can be readily explained accepting that different bacterial groups may have different responses to the presence of roots in the environment or may respond more slowly to the shifts in the environmental factors applied in the experiment (such as light and temperature or watering regimes). However, other differences require a closer look; in the $\alpha$-proteobacteria, it seems clear from the dissimilarity levels of the dendrogram (Figure 2b), that there were greater differences between rhizosphere treatments than what can be deduced from the CA plot (Figure 3b). This can be explained by the fact that the CA analysis, which aims to retain a maximum of the total variance of the samples in two dimension (Legendre and Legendre, 1998), was probably biased by the strong differences between soil and rhizosphere samples (first dimension) and by the presence of some uncommon bands in the rhizosphere control treatment at sampling date 3 and in the unplanted soil treatment at sampling date 1 (second dimension), thus 
conditioning the position of the profiles with respect to such factors, masking other possible differences present. The $\beta$-proteobacteria soil profiles derived from the GMM treatments exhibited a greater dispersion than expected from the eubacterial pattern. Since several studies (Nogales et al., 1999, 2001; our unpublished results) describe a higher than expected abundance of $\beta$-proteobacterial sequences on PCB-contaminated soils with different origins and postulate the possible implication of many $\beta$-proteobacteria on in situ natural PCB degradation, it is plausible that a GMM-mediated effect on the PCB congeners' abundances may have an effect on such bacteria, either by competition for resources or by the formation of new degradable intermediates. We find no straightforward explanation for the high dispersion of the acidobacteria soil genetic profiles at time points 1 and 2 (Figure 3e). It may be an experimental artifact since they are much more homogeneous at time points 3 and 4 , and at sampling time 1 the soil samples should be much more similar. Also, it is interesting to note that even though there seems to be an effect owing to treatment in the rhizosphere at sampling dates 3 and 4 , as observed in the CA plot, the overall dissimilarities between treatments were rather low. We have observed that the effect caused by the introduction of both class 1 and class 2 GM strains (compared with the inoculation of the wild-type strain) on native bacterial populations was limited to the rhizosphere and was variable depending on the bacterial group studied. Since the colonization abilities and the metabolism (except the PCB catabolism) of both GM strains were undistinguishable from those of the wild-type F113rif (Brazil et al., 1995; Villacieros et al., 2005), and they persisted in the environment at similar levels, we deduce that such differing effects may be a result of the activity of the introduced transgenes; reduction of local PCB concentrations and/or formation of new intermediates and their impact on sensitive populations, either because of toxicity or change of substrate availability. To our knowledge, there are no other studies dealing with the mid-term impact of GMMs designed for rhizoremediation on the native bacteria of polluted soils to compare our results with. However, in a recent study involving the use of a Commamonas sp.-alfalfa system to remediate a soil artificially polluted with 4-chloronitrobenzene (Liu et al., 2007), the structure of the eubacterial community was analyzed over a period of 15 days. In that case, observed differences between treatments in the presence and relative intensities of bands on denaturing gradient gel electrophoresis were considered the result of natural soil variability rather than from the introduction of the GM strain. Nevertheless, there is evidence from the release of biocontrol-oriented GMMs (Amarger, 2002; Viebahn et al., 2003; Johansen and Olsson, 2005) suggesting that the introduction of GMMs in the soil environment causes essentially the same effects on the native microorganisms than inoculation with the wild-type strains. They may induce, within the microbial rhizosphere populations, transient shifts in their structure and function but much less important than the differences induced by plant growth, plant species and other means of natural variability. Results of a field-release study with strain F113rif (Karlson, unpublished) showed that 4 years after its release, F113rif was still found in the rhizosphere of willows and their numbers had been reduced to $9.2 \%$ of the initial inoculation, and a similar behavior is expected for the GM strain F113L::1180. The later strain does not contain antibiotic resistance genes from the vector and thus it is suitable for in situ applications. The $b p h$ element in F113L::1180 is stable and lateral transfer of the element to a homologous recipient should occur at nondetectable frequencies in the rhizosphere (Ramos et al., 1994). The transgenes are derived from another soil microorganism, thus no new gene is added to the soil microbial community. Furthermore, the products of the trangenes are unrelated to any pathogenic or noxious factors. From the above, the potential human and environmental risk from the use of this $P$. fluorescens F113L::1180-Salix sp. system for the rhizoremediation of PCB-polluted soils seems very low. Nevertheless, a full-range field trial would be highly valuable to assess the long-term fitness of this GM strain in the environment as well as its real usefulness in the decontamination of PCB-polluted soils.

\section{Acknowledgements}

Daniel Aguirre de Cárcer was the recipient of a Comunidad de Madrid FPI scholarship. This work was funded by the Comunidad de Madrid grant GR/AMB/0084/2004, the Research Program MICROAMBIENTE-CM from the Comunidad de Madrid and by the UE project QLK3-CT2001-00101.

\section{References}

Amarger N. (2002). Genetically modified bacteria in agriculture. Biochimie 84: 1061-1072.

Barns SM, Takala SL, Kuske CR. (1999). Wide distribution and diversity of members of the bacterial kingdom Acidobacterium in the environment. Appl Environ Microbiol 65: 1731-1737.

Brazil GM, Kenefick L, Callanan M, Haro A, de Lorenzo V, Dowling DN et al. (1995). Construction of a rhizosphere pseudomonad with potential to degrade polychlorinated biphenyls and detection of bph gene expression in the rhizosphere. Appl Environ Microbiol 61: 1946-1952.

Drobnik J. (1999). Genetically modified organisms (GMO) in bioremediation and legislation. Int Biodeter Biodegr 44: 3-6.

Fedi S, Brazil D, Dowling DN, O’Gara F. (1996). Construction of a modified mini-Tn5 lacZY non-antibiotic 
marker cassette: ecological evaluation of a lacZY marked Pseudomonas strain in the sugarbeet rhizosphere. FEMS Microbiol Lett 135: 251-257.

Garland JL, Mills AL. (1991). Classification and characterization of heterotrophic microbial communities on the basis of patterns of community-level sole-carbonsource utilization. Appl Environ Microbiol 57: 23512359.

Glimm E, Heuer H, Engelen E, Smalla K, Backhaus H. (1997). Statistical comparisons of community catabolic profiles. J Microbiol Methods 30: 71-80.

Gomes NCM, Heuer H, Schonfeld J, Costa R, MendoncaHagler L, Smalla K. (2001). Bacterial diversity of the rhizosphere of maize (Zea mays) grown in tropical soil studied by temperature gradient gel electrophoresis. Plant Soil 232: 167-180.

Heuer H, Krsek M, Baker P, Smalla K, Wellington EMH. (1997). Analysis of Actinomycete communities by specific amplification of genes encoding $16 \mathrm{~S}$ rRNA and gel-electrophoretic separation in denaturing gradients. Appl Environ Microbiol 63: 3233-3241.

Holoubek I. (2001). Polychlorinated biphenyl (PCB) contaminated sites worldwide. In: Robertson, LW, Hansen, LG (eds). Recent Advances in Environmental Toxicology and Health Effects. The University Press of Kentucky: Lexington, pp 17-26.

Johansen A, Olsson S. (2005). Using phospholipid fatty acid technique to study short-term effects of the biological control agent Pseudomonas fluorescens DR54 on the microbial microbiota in barley rhizosphere. Microb Ecol 49: 272-281.

Kuiper I, Lagendijk EL, Bloemberg GV, Lugtenberg BJ. (2004). Rhizoremediation: a beneficial plantmicrobe interaction. Mol Plant Microbe Interact 17: $6-15$.

Legendre P, Legendre L. (1998). Numerical Ecology. Elsevier Science B.V.: Amsterdam, The Netherlands.

Leigh MB, Prouzová P, Macková M, Macek T, Nagle DP, Fletcher JS. (2006). Polychlorinated biphenyl (PCB)degrading bacteria associated with trees in a PCBcontaminated site. Appl Environ Microbiol 72: 2331-2342.

Liu L, Jiang CY, Liu XY, Wu JF, Han JG, Liu SJ. (2007). Plant-microbe association for rhizoremediation of chloronitroaromatic pollutants with Comamonas sp. strain CNB-1. Environ Microbiol 9: 465-473.

McFarland VA, Clarke JU. (1989). Environmental occurrence, abundance, and potential toxicity of polychlorinated biphenyl congeners: considerations for a congener-specific analysis. Environ Health Perspect 81: 225-239.

Morrissey JP, Walsh UF, O’Donnell A, Moenne-Loccoz Y, O'Gara F. (2002). Exploitation of genetically modified inoculants for industrial ecology applications. Antonie Van Leeuwenhoek 81: 599-606.
Nogales B, Moore ERB, Abraham WR, Timmis KN. (1999). Identification of the metabolically active members of a bacterial community in a polychlorinated biphenylpolluted moorland soil. Environ Microbiol 1: 199-212.

Nogales B, Moore ERB, Llobet-Brossa E, Rossello-Mora R, Amann R, Timmis KN. (2001). Combined use of $16 \mathrm{~S}$ ribosomal DNA and 16S rRNA to study the bacterial community of polychlorinated biphenyl-polluted soil. Appl Environ Microbiol 67: 1874-1884.

Porteous LA, Seidler RJ, Watrud LS. (1997). An improved method for purifying DNA from soil for polymerase chain reaction amplification and molecular ecology applications. Mol Ecol 6: 787-791.

Ramos JL, Diaz E, Dowling D, de Lorenzo V, Molin S, O'Gara $\mathrm{F}$ et al. (1994). The behavior of bacteria designed for biodegradation. Biotechnology (NY) 12: 1349-1356.

Ryslava E, Krejèík Z, Macek T, Novakova H, Mackova M. (2003). Study of PCB biodegradation in real contaminated soil. Fres Environ Bull 12: 296-301.

Safe SH. (1994). Polychlorinated biphenyls (PCBs): environmental impact, biochemical and toxic responses, and implications for risk assessment. Crit Rev Toxicol 24: 87-149.

Shannon CE, Weaver W. (1963). The Mathematical Theory of Communication. University of Illinois Press: Urbana, IL, USA.

Singer AC, Crowley DE, Thompson IP. (2003). Secondary plant metabolites in phytoremediation and biotransformation. Trends Biotechnol 21: 123-130.

Viebahn M, Glandorf DC, Ouwens TW, Smit E, Leeflang P, Wernars K et al. (2003). Repeated introduction of genetically modified Pseudomonas putida WCS358r without intensified effects on the indigenous microflora of field-grown wheat. Appl Environ Microbiol 69: 3110-3118.

Villacieros M, Whelan C, Mackova M, Molgaard J, Sanchez-Contreras M, Lloret J et al. (2005). Polychlorinated biphenyl rhizoremediation by Pseudomonas fluorescens F113 derivatives, using a Sinorhizobium meliloti nod system to drive bph gene expression. Appl Environ Microbiol 71: 2687-2694.

Villadas PJ, Martinez-Abarca F, Toro N. (2002). Polymerase chain reaction-temperature gradient gel electrophoresis requires the use of high-performance liquid chromatography-purified oligonucleotides. Anal Biochem 300: 101-103.

Weisburg WG, Barns SM, Pelletier DA, Lane DJ. (1991). $16 \mathrm{~S}$ ribosomal DNA amplification for phylogenetic study. J Bacteriol 173: 697-703.

Yee DC, Maynard JA, Wood TK. (1998). Rhizoremediation of trichloroethylene by a recombinant, root-colonizing Pseudomonas fluorescens strain expressing toluene ortho-monooxygenase constitutively. Appl Environ Microbiol 64: 112-118. 